\title{
Redução de modelos aplicado ao escoamento de Poiseuille com diferenças finitas
}

\author{
Marcello G. Teixeira Guilherme das N. Seguro* \\ Depto de Ciência da Computação, PPGI, UFRJ, \\ Caixa-postal: 68530 - Rio de Janeiro, RJ - Brasil \\ E-mail: marcellogt@dcc.ufrj.br, guilhermeseguro@ufrj.br, \\ Thomas Lewiner \\ Depto de Matemática, PUC-RJ, \\ Departamento de Matemática, Rua Marquês de São Vicente, 225, Gávea, RJ - 22451.900 \\ Email: lewiner@gmail.com
}

\section{RESUMO}

Problemas de controle ótimo para equações diferenciais parciais (EDP's) não-lineares são, em geral, difíceis de serem enfrentados numericamente, o que nos leva à necessidade de desenvolver novas técnicas. Técnicas de discretização modernas para equações diferenciais produzem modelos de simulação de grande dimensão, que exigem um alto esforço computacional para determinar soluções aproximadas. Tal fato ainda pode piorar se muitas dessas simulações são necessárias, por exemplo [3].

Para esse tipo de problema, técnicas eficientes de redução de dimensão são desejáveis. Além de algoritmos rápidos também é crucial que saibamos o quanto erramos. Os métodos para esse tipo de problema são desenvolvidos nos campos de técnicas de redução de bases (RB) para equações diferenciais parametrizadas e também no campo de redução de ordem de modelo (MOR, na sigla em inglês para Model Order Reduction) para sistemas dinâmicos.

A decomposição ortogonal própria (POD, pela sigla em inglês para Proper Orthogonal Decomposition) é um método poderoso e elegante de análise de dados que visa obter aproximações de pequenas dimensões para aproximar descrições de processos com dimensões elevadas. O método POD tem sido usado para aproximar descrições turbulentas de fluidos, vibrações estruturais, modelagens de deslocamento de insetos, processamento de imagens, análise de sinais, compressão de dados e detecção de danos [2]. Tal método é frequentemente usado com sucesso como técnica de redução de modelo para EDP's não-lineares, onde as funções base correspondem às soluções do sistema dinâmico em instantes de tempo pré-estabelecidos ou valores de parâmetros de controle, escolhidos pelo usuário, e são chamados de snapshots. Devido a uma possível dependência linear ou dependência quase linear, os snapshots não são úteis como base. Assim, uma decomposição em valores singulares (SVD, Single Value Decomposition) é necessária e as autofunções principais generalizadas são então escolhidas como base da POD.

Definimos como escoamento de Poiseuille todo escoamento laminar de fluidos viscosos que ocorram em dutos e que tenham por uma causa primária de movimento uma diferença de pressão. Nesse tipo de escoamento, o fluido é conduzido por paredes estacionárias que restringem o movimento. No presente trabalho iremos discretizar a equação de momento do fluxo por diferenças finitas obtendo assim uma matriz que descreve a velocidade e a pressão nos pontos da malha da fluido baseado em [1]. Feito isso, iremos portanto realizar o processo de redução de modelo usando o método POD descrito acima para validá-lo e obtermos uma curva de solução próxima a analítica que o escoamento de Poiseuille possui. Palavras-chave: redução de modelo, escoamento de Poiseuille, diferenças finitas, decomposição ortogonal própria

\footnotetext{
*bolsista de mestrado CENPES
} 


\section{Referências}

[1] J. R. Bodoia, J. F. Osterle, Finite difference analysis of plane Poiseuille and COuette flow developments, Appl. sci. Res, Section A, Vol 10 (1961): 265 - 276

[2] A. Chatterjee, An introduction to the proper orthogonal decomposition, Current Science, Vol. 78, no 7 (2010): 808 - 817

[3] K. Kunisch,S. Volkwein, Control of the Burgers equation by a reduced-order approach using proper orthogonal decomposition Journal of Optimization Theory and Applications 102.2 (1999): 345371. 